\title{
A comparison of two methods for prediction of response and rates of inbreeding in selected populations with the results obtained in two selection experiments
}

\author{
Valérie LOYWYCK ${ }^{\mathrm{a} *}$, Piter BIJMA $^{\mathrm{b}}$, \\ Marie-Hélène PINARD-van der LAAN ${ }^{\mathrm{a}}$, Johan van ARENDONK ${ }^{\mathrm{b}}$, \\ Etienne VERRIER ${ }^{c}$ \\ ${ }^{a}$ UMR Génétique et diversité animales, Institut national de la recherche agronomique / Institut \\ national agronomique Paris-Grignon, 78352 Jouy-en-Josas Cedex, France \\ ${ }^{\mathrm{b}}$ Animal breeding and genetics group, Wageningen University, PO Box 338, \\ 6700 AH Wageningen, The Netherlands \\ ${ }^{c}$ UMR Institut national de la recherche agronomique / Institut national agronomique \\ Paris-Grignon, 16 rue Claude Bernard, 75005 Paris, France
}

(Received 2 April 2004; accepted 10 November 2004)

\begin{abstract}
Selection programmes are mainly concerned with increasing genetic gain. However, short-term progress should not be obtained at the expense of the within-population genetic variability. Different prediction models for the evolution within a small population of the genetic mean of a selected trait, its genetic variance and its inbreeding have been developed but have mainly been validated through Monte Carlo simulation studies. The purpose of this study was to compare theoretical predictions to experimental results. Two deterministic methods were considered, both grounded on a polygenic additive model. Differences between theoretical predictions and experimental results arise from differences between the true and the assumed genetic model, and from mathematical simplifications applied in the prediction methods. Two sets of experimental lines of chickens were used in this study: the Dutch lines undergoing true truncation mass selection, the other lines (French) undergoing mass selection with a restriction on the representation of the different families. This study confirmed, on an experimental basis, that modelling is an efficient approach to make useful predictions of the evolution of selected populations although the basic assumptions considered in the models (polygenic additive model, normality of the distribution, base population at the equilibrium, etc.) are not met in reality. The two deterministic methods compared yielded results that were close to those observed in real data, especially when the selection scheme followed the rules of strict mass selection: for instance, both predictions overestimated the genetic gain in the French experiment, whereas both predictions were close to the observed values in the Dutch experiment.
\end{abstract}

selection experiments / poultry / inbreeding / genetic response / prediction methods

*Corresponding author: loywyck@inapg.fr 


\section{INTRODUCTION}

Selection programmes are mainly concerned with increasing genetic gain. Short-term progress should not, however, be obtained at the expense of the within-population genetic variability. Conservation of genetic variability should allow breeders to optimise selection programmes in the middle and long-term but also to face biological or economical problems. Selection and drift are two major factors affecting genetic variability. Several mathematical algorithms have been proposed to predict the evolution of inbreeding and/or genetic variability within selected populations of small size [2, 9, 23, 25, 27, 29, 32]. Extensive Monte Carlo simulation work has been done to investigate this evolution in a large range of situations and to compare different methods intended to jointly optimise both genetic gains and restrict the rate of inbreeding, and preserve the variability $[11,17,28]$. The analysis of real populations has mainly concerned the evolution of inbreeding and probabilities of gene origin based on pedigree information [13,18,30]. Markers have also been used to assess the evolution of genetic polymorphism [15].

Selection experiments provide the opportunity to check the validity of theoretical models to predict genetic response on a more or less long term $[5,10,16]$. Experimental lines are powerful tools because of the complete knowledge of the pedigree and the existence of control lines and since the environment is controlled. Moreover, laboratory species and some livestock species (e.g. poultry) present the twofold main advantage of short and discrete generations.

Theoretical models to predict the evolution within a small population of the genetic mean of a selected trait, its genetic variance and inbreeding have mainly been tested through Monte Carlo simulations. The purpose of this study was to compare theoretical predictions to experimental data. Two deterministic methods [27,29] will be considered, both assuming the additive infinitesimal model. Therefore, differences between theoretical predictions and experimental results can arise from differences between the true and the assumed genetic model, and from mathematical simplifications applied in the prediction methods. Two sets of experimental lines of chickens were used in this study: one undergoing true truncation mass selection and the other one undergoing mass selection with a restriction on the representation of the different families. Comparisons will be done by analysing response to selection and inbreeding. 


\section{MATERIALS AND METHODS}

\subsection{Experimental design and genetic parameters of the selected traits}

Two selection experiments on immune response in chickens have been conducted, one in the Netherlands [6] and another one in France [22]. The present study was based on the selected and control lines of these two experiments.

For the French experiment, 9 males and 44 females originating from a White Leghorn line and a commercial line were crossed in 1992. The F1 offspring were randomly crossed to produce the generation $0(n=523)$, from which four lines were derived. Three of these lines were selected for high values, according to three different criteria of immune response: antibody response 3 weeks after vaccination against the Newcastle disease virus (line 1, trait ND3), phagocytic activity at 12 weeks of age (line 2, trait PHA), and cellmediated immune response at 9 weeks of age (line 3, trait CC). There were two steps in the selection. First, the best animals were selected on the basis of their individual performance. Second, the sizes of the different half-sib families were approximately balanced except that if the performance of a given family was considered as too low, no individuals were selected from this family. As a consequence, this selection was intermediate between strict within-family and mass selection. The fourth line was a control line (line C1), with the parents being chosen at random. In each line and in each generation, 15 males and 30 females were chosen as parents of the next generation out of about 100 candidates in each sex. Mating was at random, except that full and halfsib matings were avoided. This selection design has produced 8 discrete generations (generation 1 to 8 ) which represent a total number of 6750 measured individuals.

For the Dutch experiment, 24 males and 96 females originating from two ISA Brown chicken lines were randomly crossed in 1981, to produce generation $0(n=614)$, after which selection started. Three lines were derived from generation 0: two selected lines, for high values (line $\mathrm{H}$ ) and low values (line L), and one control line (line $\mathrm{C} 2$ ). The selection criterion was the individual total antibody $(\mathrm{Ab})$ titre 5 days after injection of sheep red blood cells (SRBC). In lines $\mathrm{H}$ and $\mathrm{L}$, a strict mass selection was applied: each generation, 25 males and 50 females were selected out of about 150 candidates in each sex. In line C2, 40 males and 70 females were randomly chosen out of about 125 candidates in each sex. In each line, mating was at random except that full and half-sib matings were avoided. This selection design has produced 18 discrete generations (generation 1 to 18) which represent a total number of 17194 measured individuals. For each experiment, the heritability 
and the genetic variance in generation 0 of each of the selected traits have been previously estimated. The heritability in the base population, taking into account seven generations in the French experiment (generation 0 to 6), was estimated at $0.35( \pm 0.02)$ for ND3, $0.13( \pm 0.02)$ for PHA and $0.15( \pm 0.02)$ for CC [22]. Pinard also showed no significant correlated response and estimated genetic correlations between the three immune traits that did not differ significantly from zero [22]. Therefore, we assume these three traits to be independent. The heritability of the Ab titre, taking into account nine generations in the Dutch experiment (generation 0 to 8$)$, was estimated at $0.29( \pm 0.05)$ in line $\mathrm{H}, 0.36( \pm 0.04)$ in line $\mathrm{L}$ and $0.22( \pm 0.04)$ in line $\mathrm{C} 2$ [21].

\subsection{Data analysis}

At each generation, the cumulated response to selection in a given line was computed as the difference between the phenotypic mean in this line and the phenotypic mean in the corresponding control line, any phenotypic mean being computed over all animals born at the considered generation and measured. The global genetic gain per generation was computed as the slope of the linear regression of the observed cumulated response to selection over generations. The realised heritability was computed as the slope of the linear regression of the cumulated response to selection against the cumulated selection differential.

The individual coefficients of inbreeding were computed from the pedigree relationships using the algorithm of Van Raden [26]. In each line, from generation $t-1$ to generation $t$, the rate of inbreeding $\left(\Delta F_{t}\right)$ was computed following the classic formula (see, for instance, Falconer and Mackay [12]):

$$
\Delta F_{t}=\frac{F_{t}-F_{t-1}}{1-F_{t-1}}
$$

where $F_{t}$ is the average coefficient of inbreeding in the considered line, in generation $t$. Assuming a constant rate of inbreeding across generations, the global rate of inbreeding $(\Delta F)$, over $t$ generations, was computed as:

$$
\Delta F=1-\sqrt[t]{\left(1-F_{t}\right) /\left(1-F_{0}\right)} .
$$

\subsection{Methods of prediction}

The observed results on both inbreeding and genetic response in the selected lines were compared to the predictions given by two deterministic methods, 
one proposed by Verrier et al. [27] and hereafter referred to as the "VCF" method and the other one proposed by Woolliams and Bijma [29] and hereafter referred to as the "WB" method. Both methods assume an additive infinitesimal model with a normal distribution of the genetic and phenotypic values for the trait, random mating and true truncation selection. Prediction inputs are heritability and phenotypic variance of the trait in the base population (assumed here to be generation 0 ), and numbers of selected and candidate animals in each sex. In the present study, the genetic parameters previously estimated (see Sect. 2.1) were used. From generation $t$ to generation $t+1$, the response to mass selection $\left(R_{t}\right)$ was predicted according to the classic formula:

$$
E\left(R_{t}\right)=i \cdot h_{t}^{2} \cdot \sigma_{P_{t}}=i \cdot \sigma_{A_{t}}^{2} / \sqrt{\sigma_{A_{t}}^{2}+\sigma_{E}^{2}} .
$$

In this expression, $i$ is the intensity of selection, $h^{2}, \sigma_{A}^{2}$ and $\sigma_{P}^{2}$ are the heritability, the additive genetic variance and the phenotypic variance of the trait, respectively. The subscript $t$ refers to the generation where the selection occurs, and $\sigma_{E}^{2}$ is the environmental variance assumed to be constant over time. With the "VCF" method, the evolution of the genetic variance is predicted generation by generation, taking into account both the direct effect of selection on the between family genetic variance (the so-called Bulmer [7] effect) and the combined effects of drift and selection through inbreeding (the socalled Robertson [23] effect); see [27] for details. The global genetic gain was computed as the slope of the linear regression of the cumulated response to selection over generations. The "WB" method provides a prediction of the response to selection in an equilibrium situation, only considering the Bulmer effect. Bulmer-equilibrium genetic parameters were obtained by iteration and subsequently selection response was predicted using equilibrium parameters in the above equation.

Both methods also differ in the prediction of the evolution of the average coefficient of inbreeding. In the "VCF" method, this evolution is predicted generation by generation, on the basis of the probabilities of co-selection of full sibs, paternal half-sibs, maternal half-sibs and candidates which are not sibs, conditionally to the correlation of their values for the selection criterion. Thus VCF considers one generation of pedigree in the prediction of the rate of inbreeding (i.e. increased probabilities of co-selection through common sires and dams only, not through grandparents and more distant ancestors). Equation (2) provided a predicted global rate of inbreeding from predicted values of the average coefficient of inbreeding generation by generation.

The "WB" method provides a prediction of the overall rate of inbreeding $(\Delta F)$ based on the concept of long-term genetic contributions [31,32]. 
The long-term genetic contribution $\left(r_{k}\right)$ of an ancestor $k$, born in generation $t_{1}$, is the proportion of genes in animals born in generation $t_{2}$ deriving by descent from $k$, when $\left(t_{2}-t_{1}\right) \rightarrow \infty$. Assuming random mating, the expected rate of inbreeding per generation $(\Delta F)$ is proportional to the sum of squared contributions [32]:

$$
E(\Delta F)=\frac{1}{4} \sum_{k=1}^{n} r_{k}^{2}
$$

where $r_{k}$ is the contribution of ancestor $k$ and $n$ is the total number of parents in a generation. In the "WB" method, the long-term genetic contributions of ancestors are predicted conditionally on their breeding value and then, the rate of inbreeding is derived from these predicted long-term contributions [3]. The predicted overall rate of inbreeding enabled values of the average coefficient of inbreeding at each generation to be predicted through equation (1).

A pseudo-prediction of inbreeding generation by generation was also performed by putting the observed genetic contributions of ancestors from successive generations into equation (2). The observed genetic contributions were computed from the pedigree relationships using the probability of the gene origin approach [4]. Then, at each generation, a pseudo-predicted rate of inbreeding was calculated as proportional to the sum of squared-observed contributions to the reference population (generation 8 for the French experiment or 18 for the Dutch experiment) of individuals in the current generation.

\section{RESULTS}

\subsection{Phenotypic trend and response to selection}

Figure 1 shows the evolution of the phenotypic mean of each trait both in the line where it was selected and in the control line in the French experiment. The antibody response (ND3) was the trait showing the clearest and the most regular increase in mean under selection (in line 1). Conversely, the increase in the mean of the phagocytose activity (PHA) was low in line 2, and the mean of the cell-mediated immune response (CC) in line 3 showed the largest variation from one generation to the other. In each selected line, the respective means of the two traits which were not selected fluctuated without a significant trend (results not shown). Figure 2 shows the evolution of the phenotypic mean of antibody titre $(\mathrm{Ab})$ in the three Dutch experimental lines. The three lines followed the same fluctuations but significantly differed in their mean from generation 1 

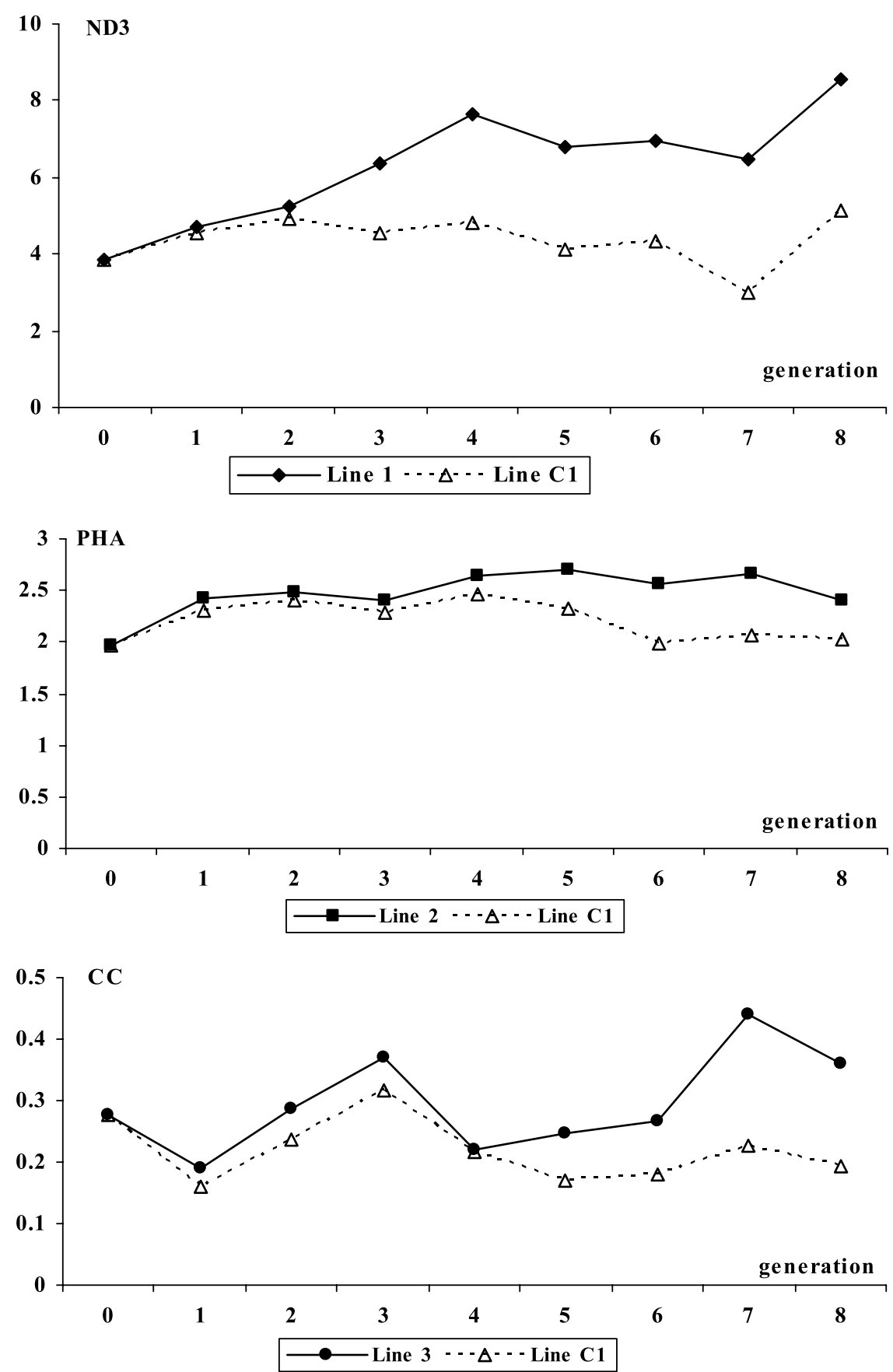

Figure 1. Evolution of the phenotypic mean of each trait in the line where it was selected and in the control line from generations 0 through 8 in the French experiment. 


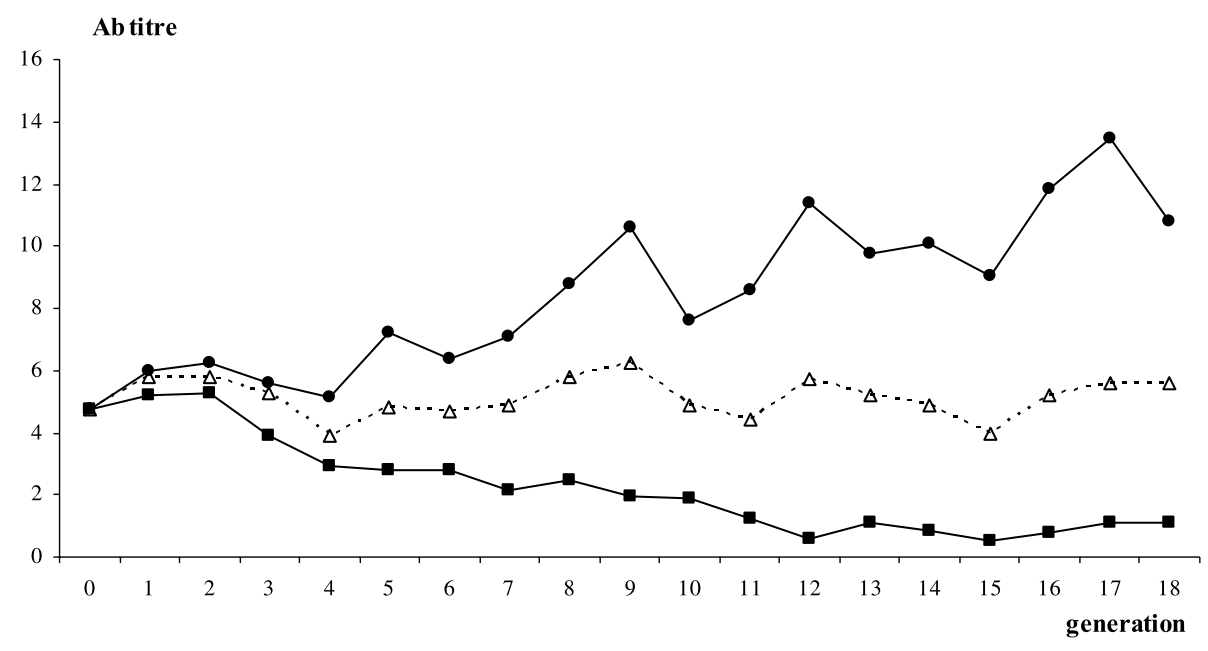

Figure 2. Evolution of the phenotypic mean of antibody $(\mathrm{Ab})$ titre in the three Dutch lines.

on for lines $\mathrm{H}$ and $\mathrm{L}$ and lines $\mathrm{L}$ and $\mathrm{C} 2$, and from generation 2 on for lines $\mathrm{H}$ and $\mathrm{C} 2$.

In the French experiment, realised heritability was found to be 0.24 for line 1, 0.02 for line 2 and 0.16 for line 3. In the Dutch experiment, realised heritability was found to be 0.13 for line $\mathrm{H}$ and 0.20 for line $\mathrm{L}$.

Figure 3 shows the evolution of the observed and predicted cumulated responses for the Dutch lines only (the French lines show a similar figure, results not shown). The curve of the observed cumulated response fluctuated: these fluctuations were not taken into account by predictions (the curves of the predicted response were almost linear). The curve of the observed response to selection in line $\mathrm{L}$ was much more irregular and slowed down for the last five generations. The curve of predicted response with the "VCF" method was steeper than the one with the "WB" method for the first generations, whereas it was the opposite in the long term.

Table I shows observation and predictions with the two methods of the global genetic gain, for the French and the Dutch experiments. Predictions with the "WB" method are higher than those with the "VCF" method. Both predictions fit the observed values in the Dutch line $\mathrm{H}$ and in the French lines 2 and 3. In the Dutch line L, the evolution of the cumulated genetic gain was not regular. However, by culling the stabilised part of the curve (generations 13 through 18), the observed global genetic gain was -0.368 and predictions 

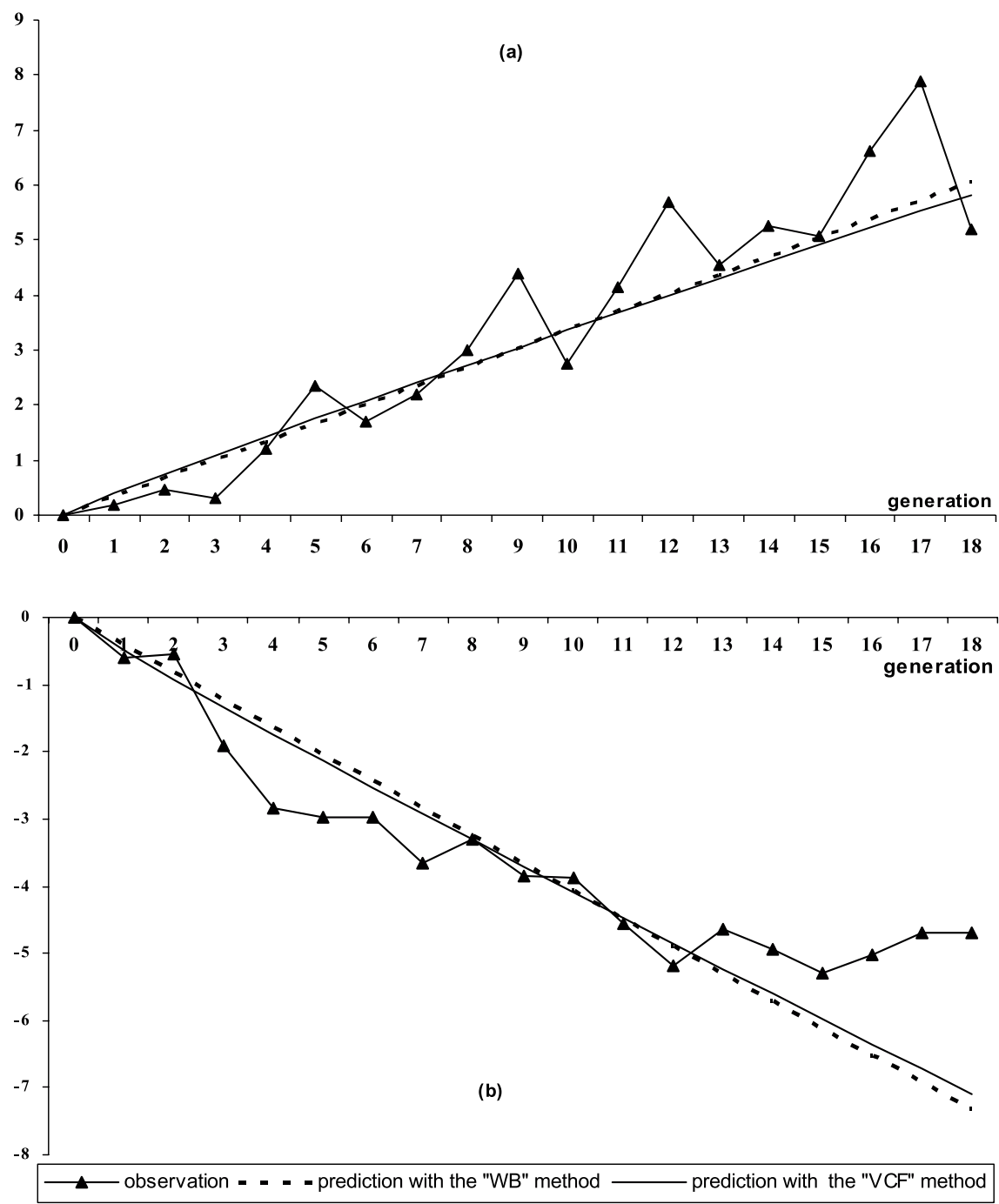

Figure 3. Evolution of the observed cumulated response to selection and the corresponding responses predicted by the two deterministic methods compared for the Dutch selected lines: line H (a) and line L (b).

were closer to the observed value with relative values of 111 for prediction with the "WB" method and 108 for prediction with the "VCF" method. In the French line 1, the observed genetic gain was overestimated by both methods of prediction. 
Table I. Observation and predictions of the global genetic gain for the French and Dutch experiments: absolute values and relative values (in italics), considering the observed value as the reference (basis $=100$ ).

\begin{tabular}{|c|c|c|c|c|c|c|c|c|c|c|}
\hline \multirow[t]{2}{*}{ Global genetic gain } & \multicolumn{6}{|c|}{ French experiment } & \multicolumn{4}{|c|}{ Dutch experiment } \\
\hline & \multicolumn{2}{|c|}{ Line 1} & \multicolumn{2}{|c|}{ Line 2} & \multicolumn{2}{|c|}{ Line 3} & \multicolumn{2}{|c|}{ Line $\mathrm{H}$} & \multicolumn{2}{|c|}{ Line $\mathrm{L}$} \\
\hline Observations & 0.483 & 100 & 0.070 & 100 & 0.0220 & 100 & 0.395 & 100 & -0.268 & 100 \\
\hline Predictions & & & & & & & & & & \\
\hline method WB & 0.643 & 133 & 0.079 & 113 & 0.0210 & 96 & 0.336 & 97 & -0.407 & 152 \\
\hline method VCF & 0.640 & 132 & 0.076 & 109 & 0.0209 & 95 & 0.321 & 98 & -0.390 & 146 \\
\hline
\end{tabular}

Table II. Observed inbreeding (\%) in the French and Dutch experiments.

\begin{tabular}{lccccccc}
\hline Inbreeding (\%) & \multicolumn{4}{c}{ French experiment } & \multicolumn{3}{c}{ Dutch experiment } \\
& Line 1 & Line 2 & Line 3 & Line C1 & Line H & Line L & Line C2 \\
\hline Generation 8 & 11.48 & 10.82 & 10.30 & 9.90 & 7.50 & 8.19 & 3.34 \\
Generation 18 & $\ldots$ & $\ldots$ & $\ldots$ & $\ldots$ & 17.77 & 16.29 & 7.26 \\
\hline
\end{tabular}

\subsection{Inbreeding}

Table II shows observed inbreeding for the French and the Dutch experiments. Inbreeding almost identically increased in the four French lines. From generations 0 through 4 , the three Dutch lines followed the same trend; however, from generations 5 through 8 , the observed inbreeding still increased for lines $\mathrm{H}$ and $\mathrm{L}$ but stabilised in the control line.

Because there was a higher number of sires and dams at each generation for the Dutch lines than for the French lines, the observed global rate of inbreeding from generations 0 through 8 was lower for the Dutch control line $(0.41 \%)$ than for the French one (1.26\%), according to the Wright equation [33]. Due to true mass selection that occurred in the Dutch selected lines and a higher number of animals in the control line, the observed global rate of inbreeding from generations 0 through 8 was two times higher for lines $\mathrm{H}$ and $\mathrm{L}(0.97 \%$ and $1.06 \%$, respectively) than for line $\mathrm{C} 2$. However, an increase of the observed global rate of inbreeding in the French experiment was partly offset by the attempt to balance family sizes after that mass selection occurred: the observed global rate of inbreeding from generations 0 through 8 was only $1.47 \%$ for line $1,1.38 \%$ for line 2 and $1.31 \%$ for line 3 .

Figure 4 shows the evolution of the observed, pseudo-predicted and predicted average coefficient of inbreeding for the Dutch selected lines only. Pseudo-predicted inbreeding was higher than predicted inbreeding with the two methods. Prediction with the "WB" method was higher than prediction with the "VCF" method. 

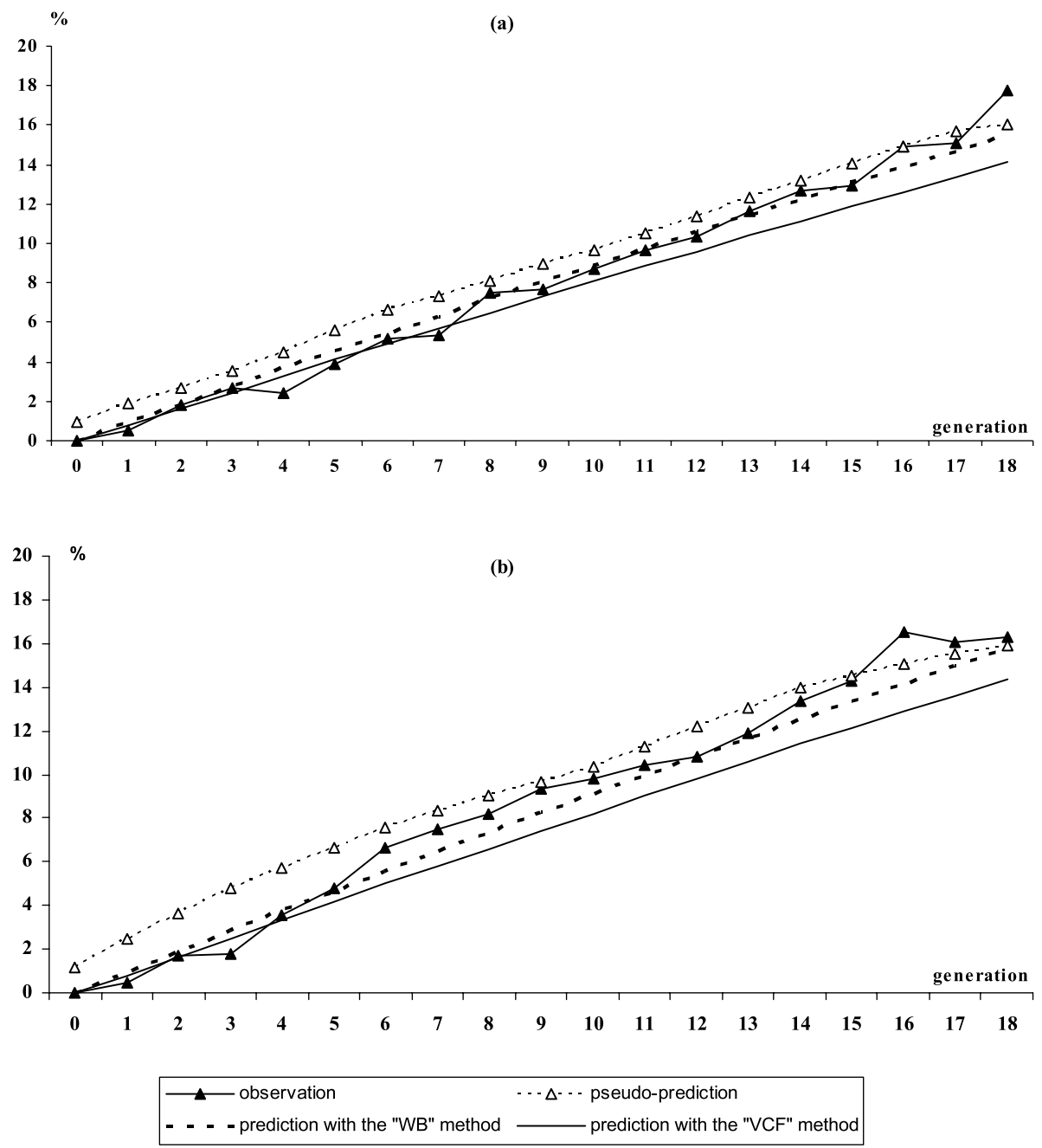

Figure 4. Evolution of observed, pseudo-predicted and predicted inbreeding for the Dutch selected lines: line H (a) and line L (b).

Table III shows observation, pseudo-prediction and predictions with the two methods of the global rate of inbreeding, for the French and Dutch experiments. Pseudo-predictions of the global rate of inbreeding are close to (except for line 2) the observed values on a short-term period (generations 0 through 8) in the French experiment as well as in the Dutch experiment, and underestimated observed values for a longer period (generations 0 through 18). Prediction with the "WB" method over-estimated the global rate of inbreeding in the French experiment whereas they were close to the observed values in 
Table III. Observation, pseudo-prediction and predictions of the global rate of inbreeding (\%) for the French and the Dutch experiments: absolute values and relative values (in italics), considering the observed value as the reference (basis $=100$ ).

\begin{tabular}{|c|c|c|c|c|c|c|c|c|c|c|c|c|c|c|c|c|c|c|}
\hline \multirow{3}{*}{$\begin{array}{l}\text { Global rate } \\
\text { of inbreeding } \\
\text { Observations }\end{array}$} & \multirow{3}{*}{$\begin{array}{l}\text { Generations } \\
0 \text { through } 8\end{array}$} & \multicolumn{8}{|c|}{ French experiment } & \multicolumn{9}{|c|}{ Dutch experiment } \\
\hline & & \multicolumn{2}{|c|}{ Line 1} & \multicolumn{2}{|c|}{ Line 2} & \multicolumn{2}{|c|}{ Line 3} & \multicolumn{2}{|c|}{ Line C1 } & \multicolumn{3}{|c|}{ Line $H$} & \multicolumn{3}{|c|}{ Line $\mathrm{L}$} & \multicolumn{3}{|c|}{ Line C2 } \\
\hline & & 1.47 & 100 & 1.38 & 100 & 1.31 & 100 & 1.26 & 100 & 0.97 & $100^{a}$ & & 1.06 & $100^{a}$ & & 0.41 & $100^{a}$ & \\
\hline & 0 through 18 & $\cdots$ & & $\cdots$ & & $\ldots$ & & $\ldots$ & & 1.08 & & $100^{b}$ & 0.98 & & $100^{b}$ & 0.42 & & $100^{b}$ \\
\hline \multirow{2}{*}{$\begin{array}{l}\text { Pseudo- } \\
\text { predictions }\end{array}$} & 0 through 8 & 1.42 & 97 & 1.19 & 86 & 1.27 & 97 & 1.36 & 106 & 0.93 & $96^{a}$ & & 1.03 & $97^{a}$ & & 0.41 & $100^{a}$ & \\
\hline & 0 through 18 & $\ldots$ & & $\ldots$ & & $\cdots$ & & $\ldots$ & & 0.91 & & $84^{b}$ & 0.89 & & $91^{b}$ & 0.39 & & $93^{b}$ \\
\hline \multirow[t]{2}{*}{ Predictions } & method WB & 1.62 & 110 & 1.42 & 103 & 1.45 & 111 & \multirow{2}{*}{$1.25^{*}$} & \multirow[t]{2}{*}{99} & 0.93 & $96^{a}$ & $86^{b}$ & 0.95 & $90^{a}$ & & \multirow{2}{*}{$0.49 *$} & \multirow{2}{*}{$120^{a}$} & \multirow{2}{*}{$117^{b}$} \\
\hline & method VCF & 1.42 & 97 & 1.32 & 96 & 1.34 & 102 & & & 0.84 & $87^{a}$ & $78^{b}$ & 0.86 & $81^{a}$ & $88^{b}$ & & & \\
\hline
\end{tabular}

* Random selection: prediction follows the Wright equation i.e. $1 /\left(8 \mathrm{~N}_{\mathrm{m}}\right)+1 /\left(8 \mathrm{~N}_{\mathrm{f}}\right)$.

${ }^{a}$ Comparison for short-term period (generations 0 through 8).

${ }^{b}$ Comparison for long-term period (generations 0 through 18). 
the Dutch experiment. On the contrary, prediction with the "VCF" method underestimated the global rate of inbreeding in the Dutch experiment whereas it was close to the observed values in the French experiment.

\section{DISCUSSION}

For both French and Dutch lines, individuals were kept and grown in the same "environment" so that they were affected by a common non-genetic year effect. However, a change in the environmental conditions occurred in the French experiment between generations 4 and 5 (use of collective cages instead of individual cages): the observed genetic mean may have been affected by this change. Thus, the observed genetic gain calculated from the evolution of the cumulated genetic gain in the French experiment has to be considered carefully.

Stabilisation of the observed genetic mean for line L could be explained by accessing a selection limit. Indeed, the aim of selection in line $\mathrm{L}$ was to decrease the $\mathrm{Ab}$ titre. The objective was reached since the phenotypic values of individuals from generations 14 through 18 were close to zero ( 0.5 at generation 15) and the Ab titre could not be negative.

No distinction was made between males and females in the Dutch experiment whereas a difference of $\mathrm{Ab}$ titre exists: females have a higher Ab titre against SRBC than males [20] therefore, the trend in Figure 1 was an average over sexes.

The two methods that were used in this study need to predict the genetic variance over time in order to give an accurate prediction of the cumulated genetic gain. Both methods seem to agree with calculations from the Dutch dataset, although the second method seems to be more accurate. However, predictions and observations were calculated assuming the additive infinitesimal model, implying that the fixation of alleles directly due to selection is ignored and the use of the infinitesimal model may be questionned for two reasons. First, the response to selection is model dependant and the additive infinitesimal model does not take into account changes in additive genetic variance due to selection [19]. Second, MHC genes are supposed to be major genes. Indeed, MHC plays an important role in the immune response and several studies have summarised the association of chicken MHC with resistance to disease $[1,8,14]$.

The predictions presented in this paper were obtained using estimated phenotypic variances and heritabilities from the same data as used in the present comparison. Consequently, prediction errors in the estimated heritability and 
phenotypic variance may be correlated to the difference between results from this experiment and average results that would have been obtained if the selection experiment had been replicated many times. Ideally, the estimation of variance components and of the "observed" response would have been based on independent data. However, long-term selection experiments in livestock are scarce and we had no independent data available for this purpose.

Pseudo-predicted inbreeding at generation $t$ is defined as being proportional to the sum of squared contributions of individuals in generation $t$ down to generation 0 . After several generations, genetic contributions of ancestors stabilise and long-term contributions are reached [32]. Therefore, contributions of individuals from the younger generations have not converged yet. Because drift would increase the loss of diversity from one generation to another, pseudopredicted inbreeding of these generations should be higher than predictions based on long-term stabilised contributions. Thus pseudo-prediction is higher than prediction with the "WB" method.

Moreover, the pseudo-predicted rate of inbreeding was over-estimated for the oldest generations. Indeed, there is no random mating due to, on the one hand, rules defined in the selection scheme (i.e. avoiding full and half-sib mating, no selfing, two sexes). On the other hand, random fluctuation of the mating system may cause deviations from Hardy-Weinberg equilibrium and therefore affect the rate of inbreeding, so that estimation should be based on the following equation:

$$
E(\Delta F)=1 / 4 \cdot \sum_{k=1}^{n}\left(1-\alpha_{I, k}\right) r_{k}^{2}
$$

where $\alpha_{I, k}$ is the deviation from Hardy-Weinberg equilibrium in individual $k$ [24]. When mating is not at random, an additional factor reduces heterozygosity: $\alpha_{I, k}$ may be seen as the correlation between alleles within individual $k$ due to mating not at random. Hence, a deviation from random mating has a higher impact on $\Delta \mathrm{F}$ when the individual in which it occurs has a higher long-term contribution.

The realised contributions of the founders could be considered as "real" long-term genetic contributions because they are calculated over a sufficiently long period to assure convergence. Thus, the contributions of founders calculated from the pedigree do not change anymore after approximately 10 generations. However, drift and inbreeding continue beyond generation 10, indicating that the contributions of founder alleles must fluctuate, despite convergence of founder contributions when calculated from the pedigree. The reason is that the contributions calculated from the pedigree are 'expected contributions given the pedigree'; truly realised contributions may deviate from pedigree 
contributions due to Mendelian sampling, which is not observable from the pedigree. Thus realised contributions of founders and long-term contributions calculated from the pedigree are not strictly equal. This discrepancy is accounted for in the derivation of the "WB" method, and predictions from it should therefore fit pseudo-predictions for the oldest generations.

\section{CONCLUSION}

This study confirmed, on an experimental basis, that modelling is an efficient approach to make useful predictions of the evolution of selected populations, despite that basic assumptions considered in the models (polygenic additive model, normality of the distribution, base population at the equilibrium, etc.) are not met in reality. The two deterministic methods yielded results that were close to real data, especially when the selection scheme followed the rules of strict mass selection.

\section{REFERENCES}

[1] Bacon L.D., Influence of the MHC on disease and productivity, Poult. Sci. 66 (1997) 802-811.

[2] Bijma P., Woolliams J.A., Prediction of rates of inbreeding in populations selected on best linear unbiased prediction of breeding value, Genetics 156 (2000) 361-373.

[3] Bijma P., van Arendonk J.A.M., Woolliams J.A., A general procedure to predict rates of inbreeding in populations undergoing mass selection, Genetics 154 (2000) 1865-1877.

[4] Boichard D., Maignel L., Verrier E., The value of using probabilities of gene origin to measure genetic variability in a population, Genet. Sel. Evol. 29 (1997) $5-23$.

[5] Bordas A., Tixier-Boichard M., Mérat P., Direct and correlated responses to divergent selection for residual food intake in Rhode Island Red laying hens, Br. Poult. Sci. 33 (1992) 741-754.

[6] Bovenhuis H., Bralten H., Nieuwland M.G.B., Parmentier H.K., Genetic parameters for antibody response of chickens to sheep red blood cells on a selection experiment, Poult. Sci. 81 (2002) 309-315.

[7] Bulmer M.G., The effect of selection on genetic variability, Am. Nat. 105 (1971) 201-211.

[8] Bumstead N., Millard B., Barrow P., Cook J.K.A., Genetic basis of disease resistance in chickens, in: Owen J.B., Axford R.F.E. (Eds.), Breeding for disease resistance in farm animals, CAB International, Melksham, UK, 1991, pp. 10-23.

[9] Caballero A., Developments in prediction of effective population size, Heredity 73 (1994) 657-679. 
[10] Clark A.G., Szumski F.M., Bell K.A., Keith L.E., Houtz S., Merriwether D.A., Direct and correlated responses to artificial selection on lipid and glycogen contents in Drosophila melanogaster, Genet. Res., 56 (1990) 49-56.

[11] Colleau J.J., Moureaux S., Briend M., Bechu J., A method for the dynamic management of genetic variability in dairy cattle, Genet. Sel. Evol. 36 (2004) 373-394.

[12] Falconer D.S., Mackay T.F.C., Introduction to quantitative genetics, 3rd edn., Longman Scientific \& Technical, Essex, UK, 1996.

[13] Huby M., Griffon L., Moureaux S., de Rochambeau H., Danchin-Burge C., Verrier E., Genetic variability of six French meat sheep breeds in relation to their genetic management, Genet. Sel. Evol. 35 (2003) 637-655.

[14] Kaufman J.F., Lamont S.J., The chicken major histocompatibility complex, in: Schook L.B., Lamont S.J. (Eds.), The major histocompatibility complex in domestic animal species, CRC Press, Boca Raton, Florida, 1996, pp. 35-64.

[15] Laval G., Chevalet C., Pitel F., Amigues Y., Bordas A., Coville J.L., TixierBoichard M., Modélisation et évaluation expérimentale de la diversité neutre ou adaptative dans le cadre de lignées sélectionnées chez la poule, in: Proceedings of $4^{\mathrm{e}}$ Colloque national du BRG, 14-16 octobre 2002, La Châtre, France, Ed. BRG, Paris.

[16] Martinez V., Bunger L., Hill WG., Analysis of response to 20 generations of selection for body composition in mice: fit to infinitesimal model assumptions, Genet. Sel. Evol. 32 (2000) 3-21.

[17] Meuwissen T.H.E., Maximizing the response of selection with a predefined rate of inbreeding, J. Anim. Sci. 75 (1997) 934-940.

[18] Moureaux S., Verrier E., Ricard A., Mériaux J.C., Genetic variability within French race and riding-horse breeds from genealogical data and blood marker polymorphism, Genet. Sel. Evol. 28 (1996) 83-102.

[19] Ollivier L., On the use of animal models in the analysis of selection experiments, Genet. Sel. Evol. 31 (1999) 135-148.

[20] Parmentier H.K., Niewland M.G., Rijke E., De Vries Reilingh G., Schrama J.W., Different response to vaccines and divergent body weight of chicken lines selected for high and low humoral responsiveness to sheep red blood cells, Avian. Dis. 40 (1996) 634-644.

[21] Pinard M.-H., van Arendonk J.A.M., Nieuland M.G.B, van der Zipp A.J., Divergent selection for immune responsiveness in chickens: estimation of realized heritability with an animal model, J. Anim. Sci. 70 (1992) 2986-2993.

[22] Pinard-van der Laan M.-H., Immune modulation: the genetic approach, Vet. Immunol. Immunopathol. 87 (2002) 199-205.

[23] Robertson A., Inbreeding in selection programs, Genet. Res. 2 (1961) 189-194.

[24] Sanchez L., Woolliams J.A., Impact of nonrandom mating on genetic variance and gene flow in populations with mass selection, Genetics 166 (2004) 527-535.

[25] Santiago E., Caballero A., Effective size of populations under selection, Genetics 139 (1995) 1013-1030.

[26] Van Raden P.M., Accounting for inbreeding and crossbreeding in genetic evaluation for large populations, J. Dairy Sci. 75 (1992) 3136-3144. 
[27] Verrier E., Colleau J.J., Foulley J.L., Predicting cumulated response to directional selection in finite panmictic populations, Theor. Appl. Genet. 79 (1990) 833-840.

[28] Verrier E., Colleau J.J., Foulley J.L., Long term effects of selection based on the animal model BLUP in a finite population, Theor. Appl. Genet. 87 (1993) 446-454.

[29] Woolliams J.A., Bijma P., Predicted rated of inbreeding in population undergoing selection, Genetics 154 (2000) 1851-1864.

[30] Woolliams J.A., Mäntysaari E.A., Genetic contributions of Finnish Ayrshire bulls over four generations, Anim. Sci. 61 (1995) 177-187.

[31] Woolliams J.A., Wray N.R., Thompson R., Prediction of long-term contributions and inbreeding in populations undergoing mass selection, Genet. Res. Camb. 62 (1993) 231-242.

[32] Wray N.R., Thompson R., Prediction of rates of inbreeding in selected populations, Genet. Res. Camb. 55 (1990) 41-54.

[33] Wright S., Evolution and the genetics of population, in: The theory of gene frequencies, Vol. 2, Univ. of Chicago, Chicago, 1969.

To access this journal online: www.edpsciences.org 
